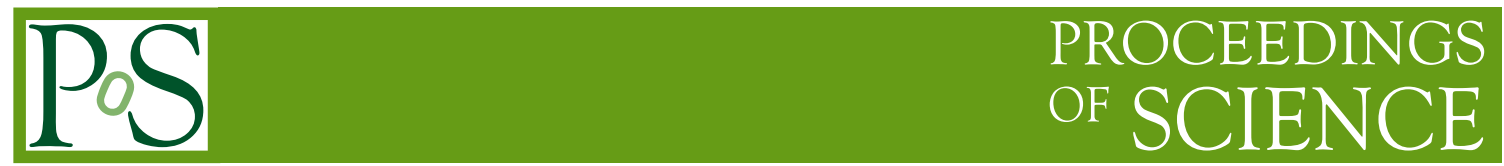

\title{
SIDR experiment status and first results
}

\section{George Bashindzhagyan*}

M.V. Lomonosov Moscow State University (RU)

E-mail: george.bashindzhagyandcern.ch

\section{Virgil Barnes}

Purdue University (IN, USA)

\section{Ephraim Fischbach}

Purdue University (IN, USA)

\section{Gagik Hovsepyan}

Yerevan Physics Institute (AM)

\section{Natalia Korotkova}

M.V. Lomonosov Moscow State University (RU)

\section{Levon Poghosyan}

Yerevan Physics Institute (AM)

\section{Nikolai Sinev}

University of Oregon (OR, USA)

\begin{abstract}
A two channel version of the SIDR (Solar Influence on Decay Rate) experiment setup is taking data in Yerevan (Armenia) and first results are presented. Two identical ${ }^{90} \mathrm{Sr}$ sources with scintillator detectors and fast electronics are permanently working at the Yerevan Physics Institute, located approximately $800 \mathrm{~m}$ above sea level. Every channel decay rate is 150 000/s. This gives us an opportunity to investigate rather fast rate changes with better than $0.1 \%$ accuracy for $10 \mathrm{~s}$ time intervals. The channels are synchronized, and the number of decays in each channel is recorded every $100 \mathrm{~ms}$. This allows us to register correlations in rate changes between the channels, and also with various environmental events, with $1 \%$ accuracy per channel within $0.1 \mathrm{~s}$.
\end{abstract}

35th International Cosmic Ray Conference - ICRC2017

10-20 July, 2017

Bexco, Busan, Korea

\footnotetext{
* Speaker.
} 


\section{Introduction}

The first experimental stage of SIDR project has been created and works now to achieve the following goals.

1. The decay rate of a few radioactive sources must be registered with very short and completely synchronized time intervals. This gives us an opportunity to observe very fast decay rate changes and their correlations between at least two independent channels and various outside events.

2. The sources must be positioned in such a way that the distance between them can be changed easily to check for any mutual influence on decay rate.

3. The accuracy of the measurements has to be very good to register minor effects. This requires rather high decay rate and fast electronics.

4. The sources must have minimal influence on the surrounding space and people to make the experiment as simple as possible. Therefore ${ }^{90} \mathrm{Sr}$ has been selected as radioactive source because it emits only electrons with maximum energy about $2.25 \mathrm{MeV}$. They can be stopped easily with 3 $\mathrm{mm}$ of steel or $15 \mathrm{~mm}$ of plastic.

\section{Experimental setup}

Cross-section of the setup is shown on Fig. 1.

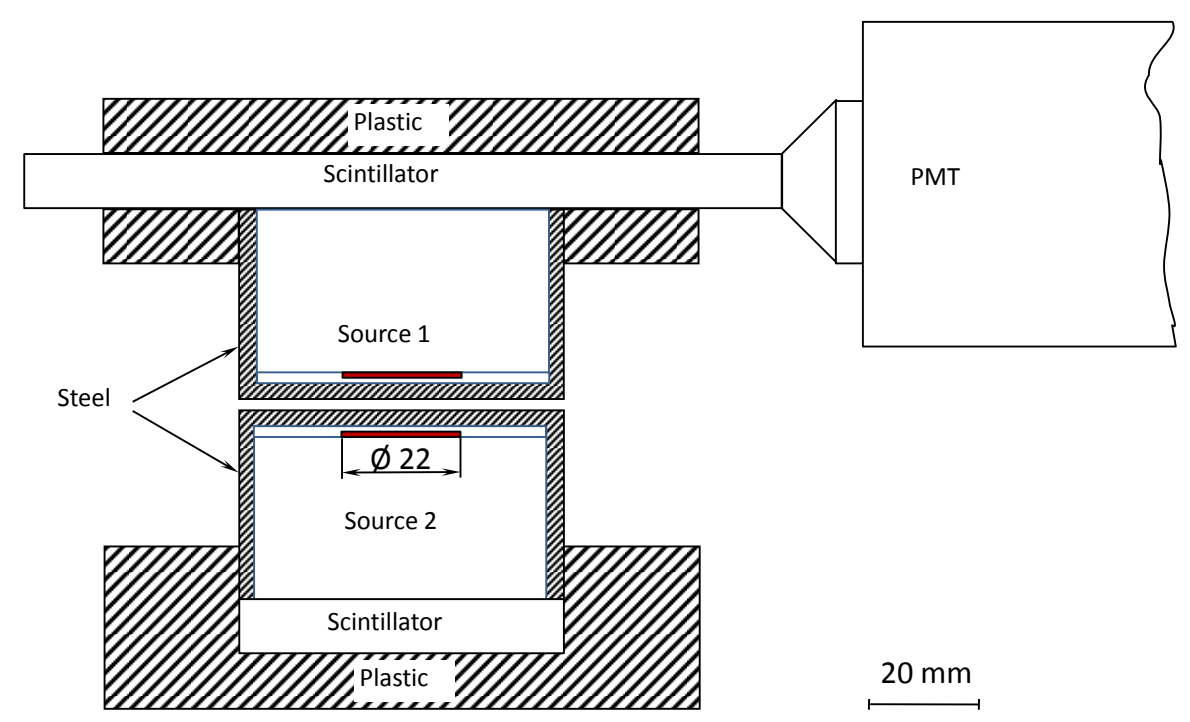

Figure 1: Experimental setup.Cross-section.

There are two separate blocks with their own electronic channels. Each of two $22 \mathrm{~mm}$ diameter flat radioactive sources is fixed on the bottom of a steel cylinder but can be easily removed to check for an absence of electronic cross-talk and other effects. The goal is to position the sources as close as possible but without a chance for neighboring electrons to cross the border. The cylinder is covered by a flat scintillator to register electron flux, and an additional layer of plastic to stop the rest. The blocks with the light collecting PMTs are positioned perpendicularly (Fig. 2.) in such a 


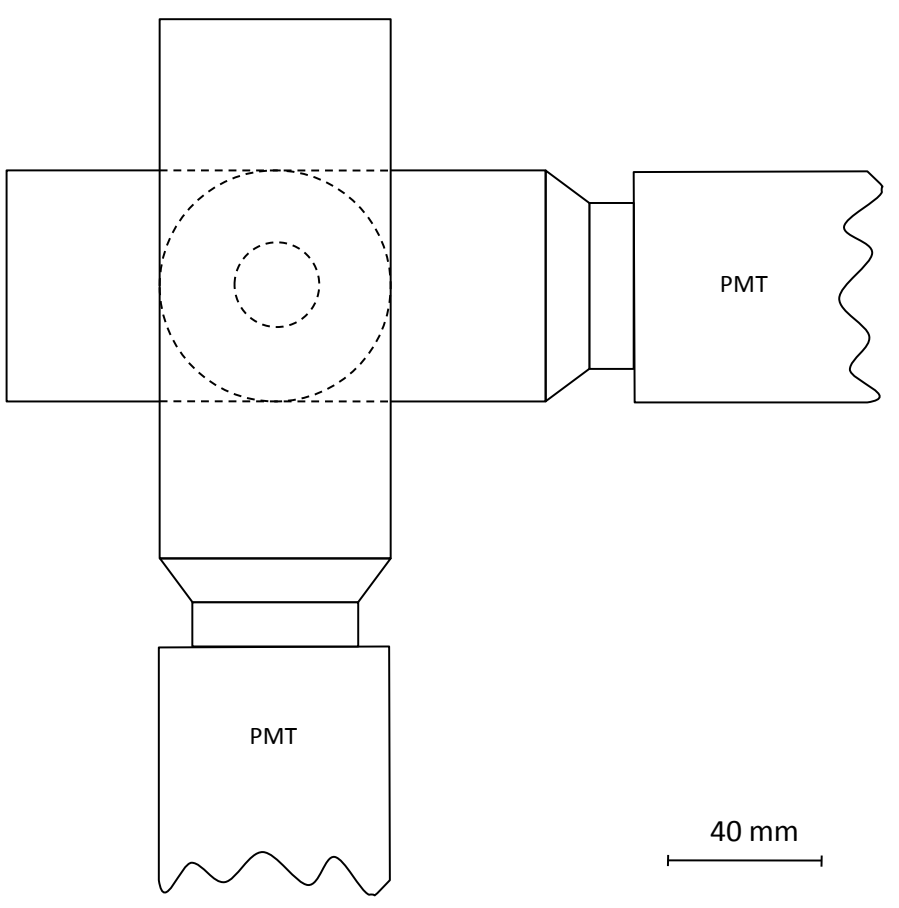

Figure 2: Experimental setup. Upper view without plastic protection.

way that the upper block can be turned out or taken off to change vertical and horizontal distances between the sources.

\section{Electronics}

Each channel has a standard set of electronic elements (Fig. 3).

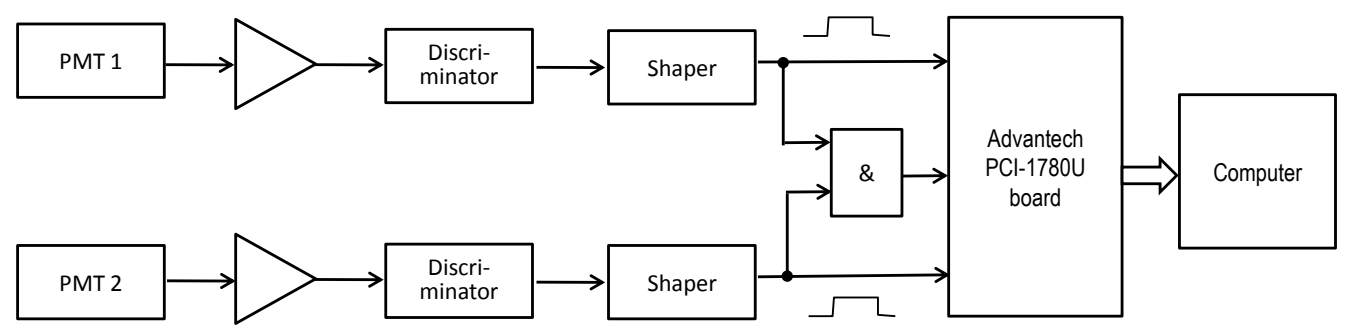

Figure 3: Electronics block diagram.

The total delay from PMT to the counter is about $25 \mathrm{~ns}$. The width of the output pulse is 250 ns, but can be tuned from 50 to $500 \mathrm{~ns}$. Special efforts have been made to stabilize the electronics but temperature stabilization is still a problem. To count the pulses a special Advantech PCI-1780U board is used. The board counts the pulses on all the inputs with synchronous start and stop every 
$100 \mathrm{~ms}$. It is very useful because fast and short rate changes can be registered. On the other hand the next 100 ms counting period starts immediately and no information is lost during long time measurements. In addition to both channels, a number of coincident output pulses from the electronic circuit is registered. For pure random process the relation between both channel rates and a number of coincidences has to be stable reflecting only the pulse width. Hence relation changes may be a signature of non-random coincidences.

\section{First results}

The plot in Fig. 4 presents the results obtained during a 6 day exposure in May 20-25, 2017. The dots show average decay number per one hour normalized for 1 second. It's visible that channel 1 is more stable and its deviations are near the calculated statistical error $\sigma \mathrm{s}= \pm 6$ counts. Channel 2 has stronger deviations and has a slope. The channel electronics are identical and most probably it reflects PMT non-stability. This is a problem which has to be solved. The third plot shows channel 1 and 2 coincidence.
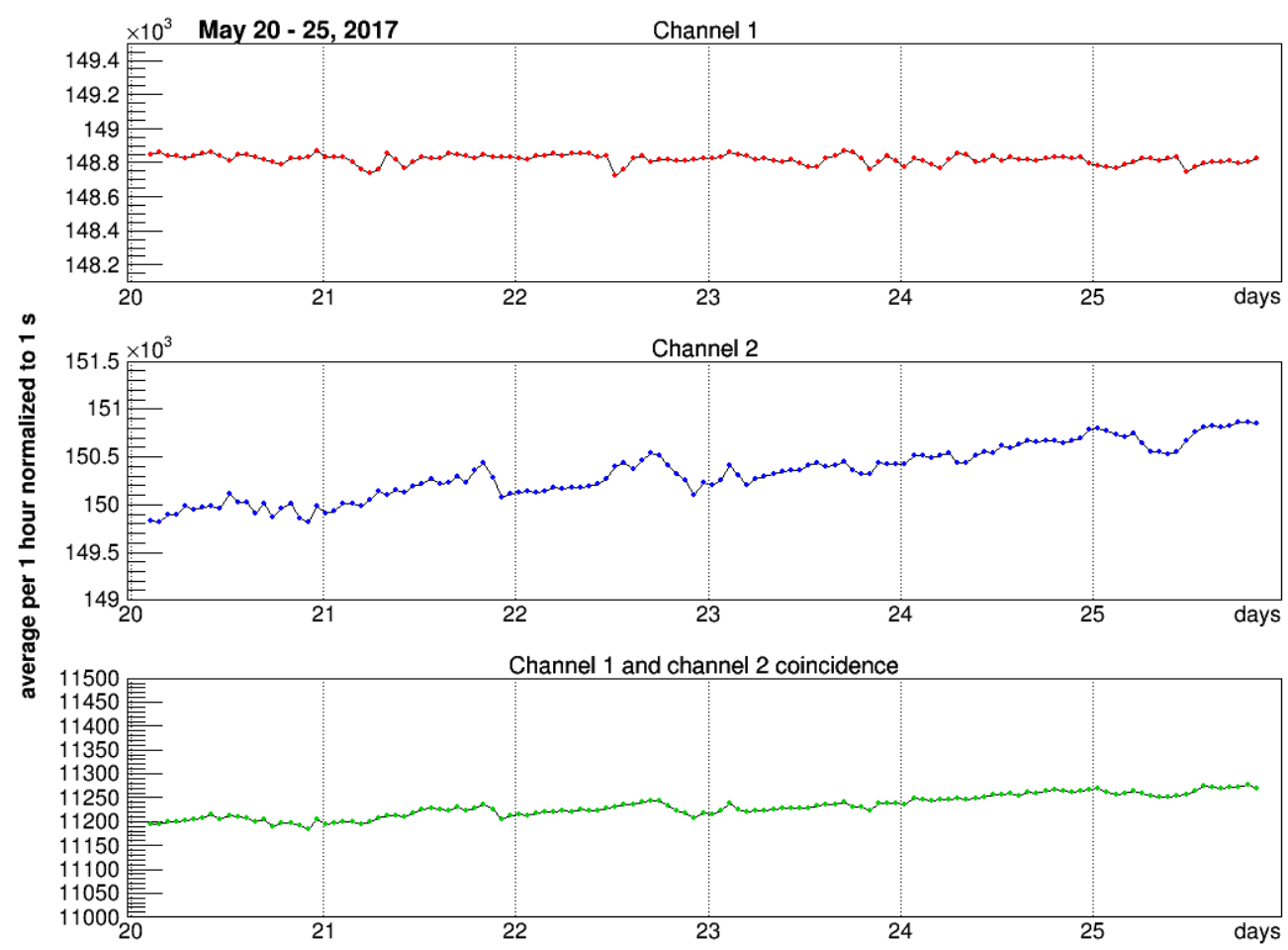

Figure 4: The results obtained during a 6 day run in May 2017.

More detailed plot with the results obtained during 1 day is presented in Fig.5.

Every dot shows the average number of decays per 10 minutes normalized to 1 second. The deviation is the same order as calculated ( \pm 16 counts) and certain rate change in time is visible. The reason of the change is unknown but it could be minor changes of the temperature.

A most interesting effect has been observed when we changed upper source position relative to lower one. To decrease the influence of slow rate changes we used short 10 minute runs changing 


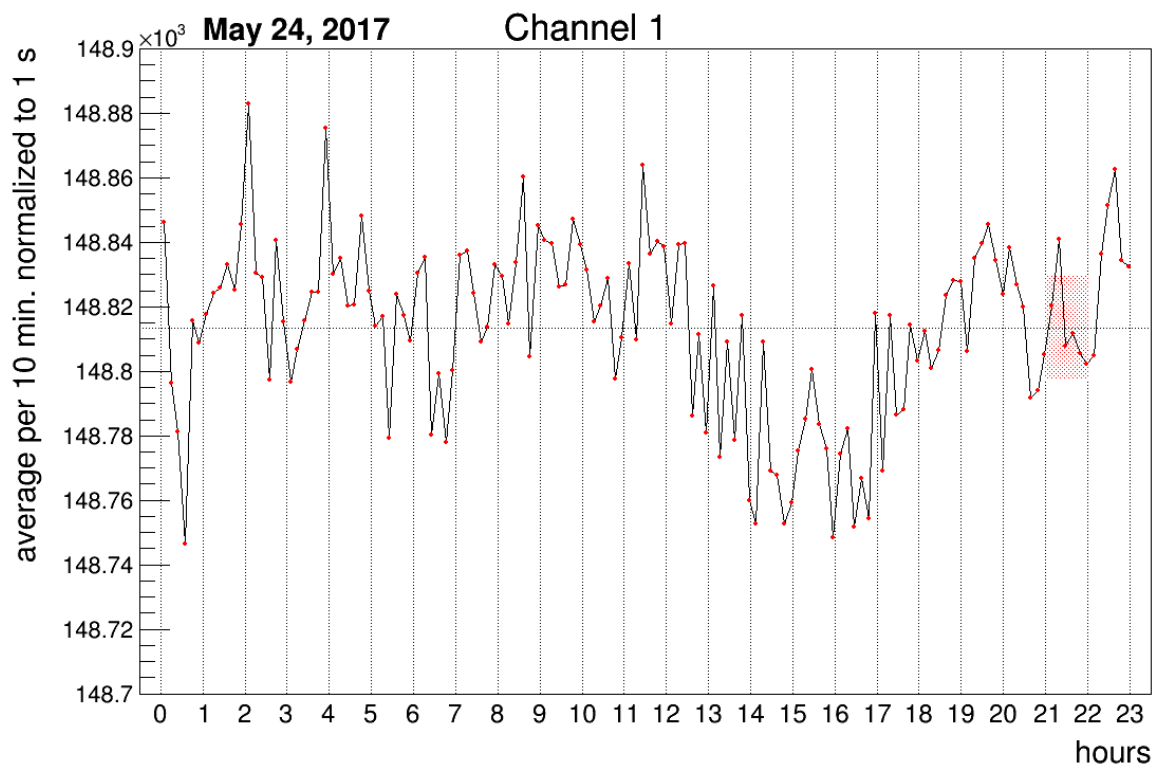

Figure 5: The results obtained during a one day run May 24, 2017.

\begin{tabular}{|c|c|}
\hline \multicolumn{2}{|c|}{ Upper source rate } \\
\hline Coupled & Separated \\
\hline counts normalized per second \\
\hline $148991 \pm 16$ & $148906 \pm 16$ \\
\hline $149036 \pm 16$ & $148986 \pm 16$ \\
\hline $149039 \pm 16$ & $148996 \pm 16$ \\
\hline $149166 \pm 16$ & $149019 \pm 16$ \\
\hline $149248 \pm 16$ & $149214 \pm 16$ \\
\hline \multicolumn{2}{|c|}{ mean value } \\
\hline $149096 \pm 7$ & $149024 \pm 7$ \\
\hline$\Delta=-72 \pm 10$ counts per second
\end{tabular}

\begin{tabular}{|c|c|}
\hline \multicolumn{2}{|c|}{ Upper source rate } \\
\hline Coupled & Separated \\
\hline counts normalized per second \\
\hline $151336 \pm 16$ & $151375 \pm 16$ \\
\hline $151278 \pm 16$ & $151386 \pm 16$ \\
\hline $151410 \pm 16$ & $151463 \pm 16$ \\
\hline $151943 \pm 16$ & $152063 \pm 16$ \\
\hline $152115 \pm 16$ & $152300 \pm 16$ \\
\hline \multicolumn{2}{|c|}{ mean value } \\
\hline $151616 \pm 7$ & $151717 \pm 7$ \\
\hline$\Delta=+101 \pm 10$ counts per second
\end{tabular}

Table 1: The difference of the average rate per second measured during 10 minutes between coupled and separated upper and lower sources.

the upper source position to opposite one after every run. Total number of counts was about $10^{8}$, statistical error $10^{4}$ and relative error $10^{-4}$. This corresponds to \pm 16 count error for normalized per 1 second data. Table 1 presents the results of 10 runs. 5 runs with "Coupled" sources when they were positioned one over the other with $8 \mathrm{~mm}$ distance and 5 runs with "Separated" sources when upper channel with source was moved apart for about $15 \mathrm{~cm}$.

The difference between the rates $\Delta$ for coupled and separated sensors is not high but visible. It's interesting that the result of a position change is opposite for upper and lower channels. The upper channel increases its rate if coupled but lower one decreases. We do not have any explanation of this result, but we plan to continue the measurements to be sure that is not a technical or electronics effect. It is necessary to say that cosmic ray flux can't give more than 5 counts per 
second and it was carefully checked.

\section{Conclusion}

The first stage of SIDR experiment is working now. The first results show that two identical channels with identical ${ }^{90} \mathrm{Sr}$ sources can work synchronously and register short term decay rate changes and change correlations between the channels as well as with outside environment. The high decay rate allows us to detect minor rate changes. One possible anomaly has been observed and needs further experimental research to confirm it or to find a logical explanation. As a next step we want to improve the existing device, especially to make it less temperature dependent. As a second step we plan to develop a new readout system with silicon sensors, to increase number of channels at least to 3 , and to position the sources in different places including underground.

\section{References}

[1] G.Bashindzhagyan, V.Barnes, E.Fischbach, G.Hovsepyan, N.Korotkova, M.Merkin, N.Sinev, A.Voronin, Solar Influence on Decay Rate (SIDR) Experiment, in proceedings of 34th International Cosmic Ray Conference, The Hague, The Netherlands, 2015 